\title{
Estudio de confiabilidad de la prueba de sialometría para flujo no estimulado en sujetos adultos clínicamente sanos
}

\section{Study of reliability of the sialometry test for non-stimulated flow in clinically healthy adult individuals}

\author{
Aitken Saavedra J¹, Maturana Ramírez A , Morales Bozo I², Hernández Ríos $\mathrm{M}^{3}$, Rojas-Alcayaga G ${ }^{4}$
}

\begin{abstract}
RESUMEN
Introducción: En personas sanas, la velocidad de flujo salival o sialometría (VFS) puede afectarse por la edad, género y ritmo circadiano. No existe evidencia de la reproducibilidad de VFS no estimulada determinada en 5 minutos, en distintos momentos del día en un mismo individuo. Objetivos: Determinar confiabilidad de VFS no estimulada medida en 5 minutos, reproducibilidad en el tiempo y relación con rango etario y género. Metodología: Se determinó VFS durante 15 minutos en 42 individuos clínicamente sanos, con una mediana de 45.5 (30-65) años, entre 9 y 11 AM durante dos mañanas y entre 3 y 5 PM durante la tarde del segundo día de medición. La saliva se colectó en tubos separados durante 5 minutos y durante los 10 minutos restantes. El peso de las muestras fue expresado en $\mathrm{ml} / \mathrm{min}$. Los valores entre los grupos de estudio, se compararon mediante test $t$ de Student, ANOVA y coeficiente de correlación de Spearman. Resultados: VFS promedio fue de $0.623 \pm 0.329$ y de $0.551 \pm$ 0.289 a los 5 y 15 minutos respectivamente $(p=0.001)$. VFS fue mayor en hombres a los 5 y 15 minutos ( $p=0.001)$. VFS en mujeres, disminuyó al aumentar la edad. No hubo diferencias en VFS a los 5 minutos $(p=0.375)$ y a los 15 minutos $(p=0.825)$, en distintos días y momentos del día, en un mismo individuo. Conclusión: VFS colectada durante 5 minutos, en un mismo individuo, presenta valores constantes en distintos días y momentos del día.
\end{abstract}

Rev. Clin. Periodoncia Implantol. Rehabil. Oral Vol. 6(1); 25-28, 2013.

Palabras clave: Flujo salival, no estimulado, sialometría, individuos sanos, adultos.

\begin{abstract}
Introduction: In healthy persons, the salivary flow rate (VFS) or sialometry can be affected by the age, the gender and the circadian rhythm. There is no evidence of the reproducibility of the non-stimulated VFS determined in 5 minutes, in different moments of the day in the same individual. Aim: To determine the reliability of the non-stimulated VFS measured in 5 minutes, its reproducibility over time and its relation with the age range and the gender. Methodology: VFS was determined for 15 minutes in 42 clinically healthy individuals, with a median age of 45.5 (30-65), between 9 and 11 AM in two mornings and between 3 and 5 PM in the evening of the second day of measurement. The saliva was collected in 2 separated pipes, one for the first 5 minutes and one for the 10 remaining minutes. The weight of the samples was expressed in $\mathrm{ml} / \mathrm{min}$. The values between the groups of study were compared by means of the Student's t-test, ANOVA and the Spearman's correlation coefficient. Results: The average VFS was of $0.623 \pm 0.329$ and of $0.551 \pm 0.289$ after 5 and 15 minutes respectively $(p=0.001)$. VFS was higher in men after 5 and 15 minutes $(p=0.001)$. VFS in women diminished as the age increased. There were no differences in VFS after 5 minutes $(p=0.375)$ and after 15 minutes $(p=0.825)$, in different days and moments of the day, in the same individual. Conclusion: VFS collected for 5 minutes in the same individual presents constant values in the different days and moments of the day. Rev. Clin. Periodoncia Implantol. Rehabil. Oral Vol. 6(1); 25-28, 2013.
\end{abstract}

Key words: Non-stimulated salivary flow, sialometry, healthy adult individuals.

\section{INTRODUCCIÓN}

La saliva tiene propiedades físico-químicas y biológicas que ofrecen protección a los tejidos de la cavidad bucal. Además, juega un papel relevante en la facilitación de procesos fisiológicos orales, como la fonación, masticación, digestión, y degustación de los alimentos. También, la saliva es un indicador del estado sistémico del individuo a través de la presencia de biomarcadores detectados en su composición ${ }^{(1-4)}$. Considerando estos aspectos, una disminución severa en la producción salival puede determinar una mala calidad de vida relacionada a la salud, así como también, constituir una condición de riesgo para el desarrollo de patologías orales tales como caries, enfermedad periodontal, queilitis angular y candidiasis ${ }^{(5-8)}$.

La secreción salival es controlada por el sistema nervioso autónomo. Se estima que la producción salival diaria oscila entre 500 y
$700 \mathrm{ml}$, con un volumen medio en la boca de $1.1 \mathrm{ml}^{(3)}$. En personas sanas, la tasa de flujo salival basal o no estimulado, puede verse afectada por factores como la edad y género del individuo en cuestión ${ }^{(9-11)}$, tamaño glandular, estado de hidratación, peso corporal, factores ambientales y factores emocionales ${ }^{(12)}$. El uso de ciertos medicamentos, así como el padecimiento de enfermedades sistémicas como diabetes e hipertensión y otras de etiología autoinmune que destruyen el estroma glandular, pueden afectar en forma reversible o definitiva, la producción de saliva ${ }^{(13-15)}$

El test de velocidad de flujo salival (VFS) o sialometría, es un procedimiento destinado a medir la cantidad de saliva que produce una persona en un tiempo determinado. Tiene la ventaja de ser de fácil implementación, de bajo costo y estar al alcance de la mayor parte de la población en riesgo ${ }^{(16)}$. Se habla de hiposalivación cuando el flujo salival se encuentra disminuido y sialorrea cuando la producción de

1. Instructor. Departamento de Patología y Medicina Oral. Facultad de Odontología, Universidad de Chile. Chile.

2. Profesor Asociado. Departamento de Ciencias Químicas y Físicas. Facultad de Odontología, Universidad de Chile. Chile.

3. Profesor Asistente. Departamento de Patología y Medicina Oral. Facultad de Odontología, Universidad de Chile. Chile.

4. Profesor Asociado. Departamento Patología y Medicina Oral. Facultad de Odontología, Universidad de Chile. Chile.

Correspondencia autor: Juan Pablo Aitken Saavedra. juanpabloaitken@gmail.com. Trabajo recibido el 04/09/2012. Aprobado para su publicación el 05/02/2013. 
saliva es excesiva ${ }^{(17,18)}$. El reconocimiento oportuno y adecuado de estos parámetros y de su severidad en distintos cuadros clínicos, es relevante para determinar tratamientos preventivos de enfermedades orales de alta prevalencia provocadas por la alteración del flujo salival| ${ }^{(5,6,18)}$. También, puede ser de utilidad para evaluar la necesidad de implementación de terapias farmacológicas o alternativas que resuelvan el trastorno de salivación correspondiente.

Si bien la sialometría es una prueba validada y muy utilizada tanto en forma clínica como en investigaciones, poco se sabe de la reproducibilidad de sus resultados a través del tiempo en un mismo individuo. Algunos estudios establecen que el horario de determinación del flujo salival se asocia con diferencias significativas en los valores obtenidos $^{(19,20)}$. Estas variaciones podrían atribuirse a los ciclos circadianos $^{(20,21)}$. Por esta razón, algunos autores recomiendan que la medición de VFS se realice en la mañana y con al menos una hora de ayuno(11,16,19). Sin embargo, no siempre es posible realizar este procedimiento en el horario sugerido, por lo que resulta de alto interés, determinar si es que existe una variación significativa en el flujo salival medido en un mismo individuo en distintos momentos del día.

Por otra parte, en distintas patologías en las que el parénquima glandular se encuentra afectado en forma significativa ${ }^{(15)}$, para definir un tratamiento, es necesario determinar si es posible estimular en estos pacientes, la secreción salival. Para ello, se debe estimar el flujo salival estimulado y no estimulado. De acuerdo a los protocolos propuestos por Navasezch en el año 1993, se establece por convención, que la medición del flujo salival no estimulado se realice recolectando la saliva durante 15 minutos, mientras que la medición del flujo estimulado, se efectúe durante 5 minutos. La posibilidad de disminuir el tiempo utilizado en determinar VFS no estimulado, podría significar un aporte respecto de la comodidad del paciente, que eventualmente permanecería menos tiempo en el sillón dental, lo que además puede ser relevante en la utilización de los recursos en servicios públicos de alto flujo de pacientes. Sin embargo, para ello se requiere validar si los valores de flujo salival no estimulado, no se ven afectados al disminuir el tiempo de recolección. Estudios que comparen los valores de flujo salival no estimulado utilizando distintos tiempos de colección, hasta nuestro conocimiento, no han sido realizados. Varios estudios recientes, en los que se determina flujo salival no estimulado, utilizan un tiempo de recolección de 5 minutos ${ }^{(22-26)}$. Sin embargo, para efectos de utilidad clínica es necesario validar su uso en esta condición de tiempo y determinar su independencia de variaciones debidas al ritmo circadiano.

En el presente estudio se pretende determinar la confiabilidad de la sialometría de flujo salival no estimulado medida en 5 minutos y su reproducibilidad en el tiempo en el mismo individuo y conforme estos resultados, establecer diferencias según rango etario y género.

\section{PACIENTES Y MÉTODOS}

\section{Muestra}

Se estimó una muestra por conveniencia de 42 individuos voluntarios. La selección fue en base a un muestreo no probabilístico de tipo intencional o por conveniencia, teniendo en cuenta que las unidades de análisis deben cumplir requisitos indispensables para la investigación (criterios de inclusión y exclusión). Este estudio se realizó en las dependencias de la Facultad de Odontología de la Universidad de Chile, conforme los principios de bioética universales de acuerdo a la declaración de Helsinki ${ }^{(27)}$. Cada persona debió firmar un acta de consentimiento informado para ser reclutado en el estudio.

\section{Criterios de Selección}

Inclusión

Individuos chilenos clínicamente sanos, de ambos sexos en el rango etario entre 30 y 70 años de edad, residentes en la ciudad de Santiago de Chile.

\section{Exclusión}

Pacientes con antecedentes de alguna enfermedad sistémica como diabetes, insuficiencia renal crónica e hipertensión arterial, en tratamiento con fármacos hiposalivantes conocidos, con enfermedades reumatológicas, irradiados en zona de cabeza y cuello, mujeres embarazadas, pacientes con enfermedades terminales, con daño neurológico o con procesos inflamatorios agudos en boca.

\section{Determinación de Flujo Salival No Estimulado}

Las muestras de saliva fueron tomadas por un único cirujanodentista, previamente entrenado, entre las 9 AM y 11 AM y entre las 3 PM y 5 PM horas conforme el protocolo descrito por Navazesh ${ }^{(16)}$. Los individuos no fumaron, no se cepillaron los dientes, ni consumieron alimentos durante una hora previa a la toma de muestra. Luego de un enjuague con agua destilada y de 5 minutos de un estado de relajación previa, se solicitó a los pacientes asumir la posición de cochero descrita por Shultz y utilizada por Navazesh ${ }^{(11,16)}$ y depositar saliva durante 5 minutos en un tubo estéril previamente rotulado. Inmediatamente y sin descontinuar la toma de muestra, la saliva total no estimulada de los siguientes 10 minutos fue colectada en un segundo tubo. El primer tubo constituyó la saliva total no estimulada de 5 minutos. Ambos tubos constituyeron la saliva total no estimulada de 15 minutos. Durante todo el procedimiento, los individuos permanecieron sentados, tranquilos y sin conversar. Este procedimiento se realizó en todos los voluntarios en la mañana de una primera sesión y en la mañana y en la tarde de una segunda sesión, realizada una semana después.

Mediante el protocolo descrito por Heintze ${ }^{(10)}$, la saliva colectada durante 5 y 15 minutos fue pesada por gravimetría asignando un peso específico de $1.005 \mathrm{~g} / \mathrm{ml}$ al fluido y el volumen total determinado, se expresó en $\mathrm{ml} / \mathrm{min}$.

\section{Análisis Estadístico}

Se realizó análisis estadístico descriptivo en base a promedio y desviación estándar para edad y flujo salival y en base a proporciones para género. Se aplicaron pruebas de normalidad para las variables cuantitativas en base a Shapiro Wilk. Se calcularon los valores para un intervalo de confianza del $95 \%$ en caso de las variables cuantitativas. Para comparar diferencias de VFS entre hombres y mujeres y entre 5 y 15 minutos, se utilizó t test. Para comparar diferencias de VFS entre mañana 1, mañana 2 y tarde, se utilizó ANOVA. Para establecer correlación entre VFS y edad, se utilizó el coeficiente de correlación de Spearman. Se aceptaron diferencias estadísticas con un nivel de significación menor a $0.05(p<0.05)$. Se ocupó el software Stata.11.

\section{RESULTADOS}

Del total de individuos, 27 eran mujeres (64.3\%) y 15 hombres (35.7\%). Un $38.1 \%$ de los individuos estaban en el rango etario de entre 30 y 39 años, un $19 \%$ en el de 40 a 49 , el $16.6 \%$ en el de 50 a 59 y un $26.2 \%$ en el de 60 a 69 años. La edad para el total de la muestra presentó una mediana de $45.5(30-65)$ años, siendo de $45(30-62)$ años y de 46 (30-65) años, para los hombres y las mujeres, respectivamente.

Los valores de VFS para el grupo total, fueron de $0.623 \pm 0.329$ a los 5 minutos y de $0.551 \pm 0.289$ a los 15 minutos ( $t$ test, $p=0.001$ ). Los valores de VFS en mujeres fueron de $0.541 \pm 0.333$ a los 5 minutos y de $0.487 \pm 0.277$ a los 15 minutos ( $t$ test, $p=0.001$ ). Los valores de VFS en hombres fueron de $0.771 \pm 0.263$ a los 5 minutos y de $0.666 \pm 0.277$ a los 15 minutos ( $t$ test, $p=0.001$ ).

La Tabla 1 muestra los valores de VFS desagregados por género, determinados a los 5 y 15 minutos, en las mañanas o en la tarde. En general, los hombres presentaron mayor VFS que las mujeres en todas las observaciones realizadas. En ambos géneros, VFS determinada a los 5 minutos fue mayor a la determinada a los 15 minutos, con la excepción de los valores obtenidos en mujeres en la mañana 1 (Tabla 1 y Figura 1).

No existe diferencia en los valores de VFS determinados a los 5 minutos (ANOVA, $p=0.375$ ) o a los 15 minutos (ANOVA, $p=0.825$ ), comparados entre mañanas distintas y entre mañana y tarde en un mismo individuo.

El coeficiente de correlación de Spearman mostró que sólo en mujeres, existe una relación inversa entre VFS y edad tanto en 5 como en 15 minutos $(p<0.05)$. En hombres en cambio, el flujo salival no mostró variaciones en relación a la edad. 
Tabla 1. VFS no estimulado según género y tiempo de determinación.

\begin{tabular}{|c|c|c|c|c|c|c|}
\hline \multirow{2}{*}{} & \multicolumn{2}{c|}{$\begin{array}{c}\text { VFS } \\
\text { 5 minutos }\end{array}$} & \multicolumn{2}{c|}{$\begin{array}{c}\text { VFS } \\
\text { 15 minutos }\end{array}$} & \multirow{2}{*}{ p value } \\
\cline { 2 - 6 } & Promedio & DS & Promedio & DS & \\
\hline \multirow{4}{*}{ Mañana 1 } & Hombres & 0.773 & 0.255 & 0.704 & 0.251 & 0.029 \\
\cline { 2 - 6 } & Mujeres & 0.517 & 0.276 & 0.490 & 0.262 & 0.065 \\
\cline { 2 - 6 } & Total & 0.608 & 0.293 & 0.566 & 0.275 & 0.007 \\
\hline \multirow{3}{*}{ Mañana 2 } & Hombres & 0.757 & 0.342 & 0.631 & 0.336 & 0.001 \\
\cline { 2 - 6 } & Mujeres & 0.553 & 0.364 & 0.503 & 0.280 & 0.021 \\
\cline { 2 - 6 } & Total & 0.626 & 0.366 & 0.549 & 0.303 & 0.001 \\
\hline \multirow{3}{*}{ Tarde } & Hombres & 0.783 & 0.187 & 0.663 & 0.248 & 0.001 \\
\cline { 2 - 6 } & Mujeres & 0.552 & 0.364 & 0.468 & 0.297 & 0.001 \\
\cline { 2 - 6 } & Total & 0.635 & 0.329 & 0.537 & 0.293 & 0.001 \\
\hline
\end{tabular}

DS = Desviación estándar.

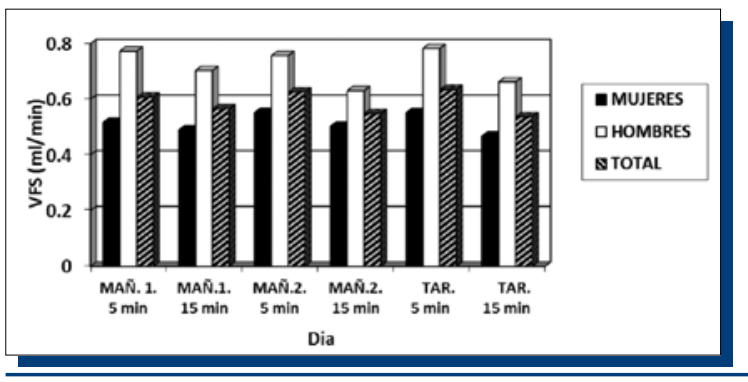

Figura 1. VFS no estimulado según género a los 5 y 15 minutos en mañanas y tarde.

\section{DISCUSIÓN}

En el presente estudio determinamos la confiabilidad de la sialometría de flujo salival no estimulado medida en 5 minutos y su reproducibilidad en el tiempo en el mismo individuo. Conforme a estos resultados, establecimos diferencias según rango etario y género.

Nuestros resultados mostraron mayor VFS determinada a los 5 minutos en todas las mediciones. Hasta nuestro conocimiento, no existen estudios que comparen la determinación de VFS no estimulado entre 5 y 15 minutos. Sin embargo, podemos relacionar nuestros resultados con estudios realizados en individuos sanos, a quienes se les suministró pilocarpina y que luego de medir flujo salival durante 40 minutos, se estableció el máximo de VFS alrededor de los 10 minutos, valor que cae a los 15 minutos y tiende a estabilizarse hasta completar la medición ${ }^{(28)}$. Un estudio de VFS estimulado, establece que la parafina en boca aumenta la velocidad del flujo, pero que este fenómeno no constituye un estado permanente ${ }^{(29)}$. Otros estudios señalan que la VFS no es constante y tiende a experimentar picos de exacerbación ${ }^{(19,20)}$. Si bien nuestro trabajo se realizó con mediciones de saliva no estimulada, puede que exista un pico de velocidad de flujo salival que luego se estabiliza. Un estudio que evalúa el efecto del estrés en la VFS, también establece que luego de 10 minutos de secreción salival, ésta comienza a decrecer para pasar a un estado de estabilización de producción ${ }^{(22)}$. Aunque en nuestro estudio VFS fue mayor a los 5 minutos que a los 15 , los valores en ambos tiempos de medición, se correlacionaron de la misma forma con las variables de género y edad. Estudios adicionales son necesarios para determinar si la medición de VFS a los 5 minutos permitiría identificar inequívocamente a individuos con hiposialia. Además, dado que en la mayoría de los estudios se determina VFS no estimulado en 5 minutos, se hace necesario replantear los protocolos para su medición, de modo que los resultados obtenidos en distintos estudios, puedan ser comparados.

Existe suficiente evidencia respecto a que el horario de medición influye en el valor de VFS. Un estudio observó que la sialometría medida en ayunas, presenta en promedio, una disminución de entre 0.04 y $0.05 \mathrm{ml} / \mathrm{min}$ en comparación a la que se mide al medio día, diferencia que se atribuye no sólo al ritmo circadiano, sino que también, al mayor grado de hidratación que otorga la alimentación matinal. El mismo estudio señala que un gran número de sujetos muestra hiposalivación a las 7:30 $\mathrm{AM}$ pero no a las 11:30 $\mathrm{AM}^{(19)}$. Estos resultados se asocian a lo descrito por otros estudios donde se señala que la menor producción salival ocurre durante las horas de sueño, por lo que el pico de sequedad bucal ocurre antes de despertar ${ }^{(17,19,20)}$. Nuestro estudio no evidenció diferencias significativas en la VFS medida en distintos horarios, lo que podría hacernos suponer, un mayor grado de hidratación de las personas evaluadas ya que estaban en un ayuno de sólo una hora. Estos resultados sugieren que de acuerdo el protocolo utilizado, el horario de medición no parece ser un factor determinante en los valores de VFS. De acuerdo a esto, sería posible medir VFS tanto en la mañana como en la tarde, obteniendo resultados similares. Un estudio adicional, incluyendo un mayor número de individuos de distintos grupos etarios, permitiría avalar esta hipótesis.

En nuestro estudio, las mujeres presentaron menor VFS en comparación con los hombres, independiente del tiempo de medición y momento del día escogido para realizarla. Nuestros resultados son coincidentes con los obtenidos en otros estudios que describen un fenómeno similar ${ }^{(9,10,23,24,30)}$. En algunos de ellos, se asocia la menor VFS en mujeres a fluctuaciones hormonales descritas durante la pubertad, menstruación y el embarazo ${ }^{(23,24)}$. Sin embargo, ninguna de las mujeres incluidas en nuestro estudio estaba embarazada ni era adolescente. Existe suficiente evidencia que señala que durante la menopausia, se reduce la VFS, efecto que se compensa con las terapias farmacológicas de reemplazo ${ }^{(25)}$. Otro estudio ha observado menor VFS en mujeres menores de 30 años, asociado al sobrepeso. Esto podría deberse a que el contenido de los nutrientes, ricos en sodio y grasa, provocarían una disfunción glandular ${ }^{(12)}$. Si bien este fenómeno también se describe en hombres, su efecto es mayor en mujeres. Sin embargo, el peso corporal de los individuos, no fue considerado en nuestro estudio. Otros autores, han relacionado la diferencia de VFS con el menor tamaño de las glándulas salivales descrito en mujeres ${ }^{(4,9,30)}$. Estos resultados sugieren la necesidad de definir parámetros normales de salivación diferentes para ambos géneros.

En relación a la disminución de VFS asociada al envejecimiento, en nuestro estudio sólo las mujeres presentaron este fenómeno, lo que concuerda con los resultados de varios estudios ${ }^{(9,23,24,30)}$. Algunos autores lo atribuyen a los cambios hormonales asociados con el envejecimiento que podrían alterar el funcionamiento normal de glándulas salivales ${ }^{(23,30)}$. Otros estudios describen una disminución de VFS en hombres después de los 70 años $^{(31)}$, a diferencia de las mujeres, en que este fenómeno se describe desde edades más tempranas. En nuestro estudio, ningún individuo tenía más de 70 años, lo que explicaría la falta de asociación entre VFS y edad en hombres. También, se ha relacionado el menor VFS en adultos mayores, con el hecho que la gran mayoría de estos individuos, consume algún fármaco hiposalivante

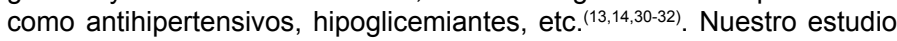
incluyó a individuos clínicamente sanos y que no consumieran este tipo de fármacos.

Los resultados de nuestro estudio sustentan la idea que VFS no estimulado, puede ser determinado, obteniendo resultados confiables, a distintas horas del día y utilizando sólo 5 minutos en su medición, lo que simplificaría significativamente el diseño de los estudios en se requiere determinar parámetros salivales.

\section{AGRADECIMIENTOS}

A Fabiana Llanos y Fernanda Díaz.

\section{CONFLICTOS DE INTERÉS}

No existe relación comercial alguna o financiamiento para la realización de este estudio. 


\section{REFERENCIAS BIBLIOGRÁFICAS}

1. Llena-Puy $C$. The role of saliva in maintaining oral health and as an aid to diagnosis. Med Oral Patol Oral Cir Bucal, 2006; 11: E449-455.

2. Streckfus C, Bibler L. Saliva as a diagnosis fluid. Oral Diseases, 2002; 8: 69-76. 3. Dodds MW, Jonson DA, Yeh CK. Health benefits of saliva: A review. J Dent, 2005; 33: 223-233.

4. Malamud D. Salivary diagnostics: The future is now. J Am Dent Assoc, 2006 137: 284-286.

5. Ergun S, Cekici A, Topcuoglu N, Migliari DA, Külekçi G, Tanyeri H, Isik G. Ora status and candida colonization in patients with Sjögren's syndrome. Med Oral Patol Oral Cir Bucal, 2010 Mar 1; 15(2): E310-315.

6. Hibino K, Samaranayake LP, Hägg U, Wong RW, Lee W. The role of salivary factors in persistent oral carriage of candida in humans. Arch Oral Biol, $2009 \mathrm{Jul}$ 54(7): 678-683

7. Galgut $\mathrm{P}$. The relevance of $\mathrm{pH}$ to gingivitis and periodontitis. J Int Acad Periodontol, 2001; 3(3): 61-67.

8. Mungia R, Cano SM, Johnson DA, Dang H, Brown JP. Interaction of age and specific saliva component output on caries. Aging Clin Exp Res, 2008 Dec; 20(6): 503-508.

9. Percival RS, Challacombe SJ, Marshi PD. Flow rates of resting whole and stimulated parotid saliva in relation to age and gende. J Dent Res, 1994; 73(8): 1416-1420

10. Heintze U, Birkhed D, Bjornhd. Secretion rate and buffer effect of resting and stimulated whole saliva as a function of age and sex. Swed Dent J, 1983; 7: 227 238.

11. Navazesh M, Mulligan RA, Kipnis V, Denny PA, Denny PC. Comparison of whole saliva flow rates and mucin concentrations in healthy caucasian young and aged adults. J Dent Res, 1992: 71(6): 1275-1278.

12. Flink $H$, Bergdahl $M$, Tegelberg A, Rosenblad A, Lagerlöf $F$. Prevalence of hyposalivation in relation to general health, body mass index and remaining teeth in different age groups of adults. Community Dent Oral Epidemiol, 2008 Dec; 36 (6): 523-531.

13. Field EA, Fear S, Higham SM, Ireland RS, Rostron J, Willetts RM, Longman LP. Age and medication are significant risk factors for xerostomia in an english population, attending general dental practice. Gerodontology, $2001 \mathrm{Jul} ; 18(1)$ : 21 24.

14. Leal SC, Bittar J, Portugal A, Falcão DP, Faber J, Zanotta P. Medication in elderly people: Its influence on salivary pattern, signs and symptoms of dry mouth Gerodontology, 2010 Jun; 27(2): 129-133.

15. Da Silva Marques DN, da Mata AD, Patto JM, Barcelos FA, de Almeida Rato Amaral JP, de Oliveira MC, Ferreira CG. Effects of gustatory stimulants of salivary secretion on salivary $\mathrm{pH}$ and flow in patients with Sjögren's syndrome: A randomized controlled trial. J Oral Pathol Med, 2011 Nov; 40(10): 785-792.

16. Navazesh M. Methods for collecting saliva. Ann N Y Acad Sci, 1993; 694: 72-77. 17. Streckfus C, Bibler L. Saliva as a diagnosis fluid. Oral Diseases, 2002; 8: 69-76.
18. Dodds MW, Jonson DA, Yeh CK. Health benefits of saliva: A review. J Dent 2005; 33: 223-233.

19. Flink H, Tegelberg A, Lagerlöf F. Influence of the time of measurement of unstimulated human whole saliva on the diagnosis of hyposalivation. Arch Oral Biol, 2005 Jun; 50(6): 553-559.

20. Zheng L, Seon YJ, McHugh J, Papagerakis S, Papagerakis P. Clock genes show circadian rhythms in salivary glands. J Dent Res, 2012; 91(8): 783-788.

21. Dawes C, Ong BY. Circadian rhythms in the flow rate and proportional contribution of parotid to whole saliva volume in man. Arch Oral Biol, 1973; 18(9): 1145-1153.

22. Sugimoto K, Kanai A, Shoji N. The effectiveness of the Uchida-Kraepelin test for psychological stress: An analysis of plasma and salivary stress substances. Biopsychosoc Med, 2009; (3): 3-5.

23. Mirzaii-Dizgah I, Agha-Hosseini F. Unstimulated whole saliva parathyroid hormone in postmenopausal women with xerostomia. J Contemp Dent Pract, 2011 May 1; 12(3): 196-199.

24. Eliasson L, Carlen A, Laine M, Birkhed D. Minor gland and whole saliva in postmenopausal women using a low potency oestrogen (oestriol). Arch Oral Biol 2003; 48: 511-517.

25. Laine M, Leimola-Virtanen R. Effect of hormone replacement therapy on salivary flow rate, buffer effect and $\mathrm{pH}$ on perimenopausal and postmenopausal women. Arch Oral Biol, 1996; 41: 91-96.

26. Sawair FA, Ryalat S, Shayyab M, Saku T. The unstimulated salivary flow rate in a jordanian healthy adult population. J Clin Med Res, 2009 Oct; 1(4): 219-225 27. De Roy PG. Helsinki and the declaration of Helsinki. World Med J, 2004; 50(1) 9-11.

28. Davies AN, Singer JM. A comparison of artificial saliva and pilocarpine in radiation induced xerostomia. J Laryngol Otol, 1994 Aug; 108(8): 663-665.

29. Laine M, Pienihäkkinen K, Leimola-Virtanen $\mathrm{R}$. The effect of repeated sampling on paraffin-stimulated salivary flow rates in menopausal women. Arch Oral Biol 1999 Jan; 44(1): 93-95.

30. Lukacs JR, Largaespada LL. Explaining sex differences in dental caries prevalence: Saliva, hormones, and "life-history" etiologies. Am J Hum Biol, 2006 18: $540-555$

31. Smidt D, Torpet LA, Nauntofte B, Heegaard KM, Pedersen AM. Associations between labial and whole salivary flow rates, systemic diseases and medications in a sample of older people. Community Dent Oral Epidemiol, 2010 Oct; 38(5): 422-435

32. Campbell NL, Boustani MA, Skopeljia EN, Gao S, Unverzagt FW, Murray MD Medication adherence in older adults with cognitive impairment: A systematic evidence-based review. Am J Geriatr Pharmacother, 2012 Jun; 10(3): 165-177. 